\title{
Core-stable rings in auctions with independent private values*
}

\author{
Omer Biran and Françoise Forges ${ }^{\dagger}$
}

April 2010 (first version: November 2009)

\begin{abstract}
We propose a semi-cooperative game theoretic approach to check whether a given coalition is stable in a Bayesian game with independent private values. The ex ante expected utilities of coalitions, at an incentive compatible (noncooperative) coalitional equilibrium, describe a (cooperative) partition form game. A coalition is core-stable if the core of a suitable characteristic function, derived from the partition form game, is not empty. As an application, we study collusion in auctions in which the bidders' final utility possibly depends on the winner's identity. We show that such direct externalities offer a possible explanation for cartels' structures (not) observed in practice.
\end{abstract}

Keywords: Auctions, Bayesian game, collusion, core, partition function game.

JEL classification: C71, C72, D44

*We are grateful to Bernard Lebrun and Ron Holzman for extremely valuable suggestions. We also acknowledge insightful comments from Francis Bloch, participants of the Game Theory Festival of Stony Brook (July 2009) and participants of seminars in Berlin (Free University and Humboldt University), Paris (MSE and "séminaire parisien de théorie des jeux") and Warwick.

${ }^{\dagger}$ CEREMADE and LEDa, Université Paris-Dauphine, Place du Maréchal de Lattre de Tassigny, 75775 Paris cedex 16. 


\section{Introduction}

Collusion in auctions is mostly studied as a mechanism design problem for a given ring (see, e.g., Graham and Marshall (1987), Mailath and Zemsky (1991) and McAfee and McMillan (1992) for early references and Marshall and Marx (2007) for a recent one). This framework imposes individual participation constraint to every member of the ring. In second price auctions with independent private values, Mailath and Zemsky (1991) further consider participation constraints for all subrings of any potential ring. In this particular framework, equilibria in weakly dominant strategies considerably limit the strategic externalities that coalitions might incur. Mailath and Zemsky (1991)'s analysis does not extend if equilibria in weakly dominant strategies do not exist, e.g., in the case of common values (see Barbar and Forges (2007)). In this paper, we keep the assumption of independent private values but except for that, allow for an arbitrary auction game. We ask whether a given coalition is stable, in the sense that no subgroup of players would like to leave it. Such collective participation constraints are traditionally captured by core-like solution concepts. However, two difficulties arise when trying to define the core of an arbitrary auction game, or, more generally, a Bayesian game.

A first difficulty, which already appears under complete information, is that every coalition faces strategic externalities, so that it must make conjectures on the behavior of the players who are outside the coalition. To solve this difficulty, Aumann (1961) introduced the $\alpha$ - characteristic function, which measures the worth of a coalition in a strategic form game as the amount that it can guarantee whatever the complementary coalition does. However, the corresponding core, namely the $\alpha$-core, can be criticized on the grounds that it involves incredible threats from the complementary coalition. As a remedy, Ray and Vohra (1997) and Ray (2007) construct a partition form game (as defined by Lucas and Thrall (1963)) in which, given a partition of the players, coalitions evaluate their worth at a Nash equilibrium of an auxiliary game between the coalitions. We extend Ray (2007)'s coalitional equilibrium to games with incomplete information and construct a partition form game from the noncooperative Bayesian game which models the auction. We then apply a notion of core for partition form games, the core with "cautious expectations" (see Hafalir (2007)). Under complete information, it is included in the $\alpha$-core.

In a coalitional equilibrium of a Bayesian game, it is understood that the 
strategy of every coalition is a function of its members' private information. The description of the previous paragraph hides a second difficulty, which is specific to incomplete information: every coalition faces incentive constraints. We establish (in proposition 1 and its corollary) that, in a class of Bayesian games which includes standard auctions (namely, games with independent private values and quasi-linear utilities), this difficulty can be ignored: every coalitional equilibrium can be made incentive compatible. More precisely, coalitional equilibria are "first best" solutions, in which every coalition plays a best reply to the strategies outside the coalition, as if information sharing was not an issue. We construct an incentive compatible revelation mechanism for the coalition, which involves exactly balanced monetary transfers among its members and achieves the "first best" reply of the coalition. The fact that the coalition maximizes its payoff given strategies in the complementary coalition is crucial to our construction, in particular, in the formulation of incentive constraints.

In order to associate a partition form (cooperative) game to a (noncooperative) Bayesian game, we assume that coalitions can commit to an incentive compatible mechanism at the ex ante stage, i.e., before their members get their private information. This assumption first requires that an ex ante stage can be identified, which is true in many economic applications, like auctions, in which private information reduces to the value of some parameter, like a valuation or a cost. According to empirical data (see, e.g., Porter and Zona (1993), Pesendorfer (2000)), bidding rings often consist of wellidentified groups (e.g., "incumbents", as opposed to "newcomers") whose characteristics do not depend on particular information states. Such bidding rings typically form at an early stage. For instance, local suppliers may be aware that a procurement auction will take place and consider to collude before the precise project specifications are published. At the time they commit to a collusion mechanism, they do not figure out their exact valuations, i.e., the costs incurred by the project.

The ex ante formation of rings is assumed explicitly in Bajari (2001), Marshall et al. (1994) and Waehrer (1999). In these papers, ring mechanisms are investigated within an a priori given partition of the bidders. The partition itself does not depend on the bidders' private information, which reflects the ex ante formation of the rings. To the best of our knowledge, interim formation of rings has only been investigated to a limited extent, e.g., in Graham and Marshall (1987), McAfee and McMillan (1992), Caillaud and Jehiel (1998) and Marshall and Marx (2007). These papers focus 
on an ex ante given bidding ring, the grand coalition for instance, and formulate interim participation constraints for the individual members of the ring. More precisely, every member of the ring can decide to leave the ring once he knows his private information. The precise form of the participation constraints depends on the reaction of ring members when one of them leaves the ring. Mailath and Zemsky (1991) start with the latter model but restrict themselves on ex ante expected payoffs when studying the stability of rings. Being interested in the participation constraints of coalitions, rather than individuals, we assume, to keep the analysis tractable, that coalitions can commit to an incentive compatible mechanism ex ante, as in Forges and Minelli (2001) and Forges, Mertens and Vohra (2002). These papers introduce the notion of ex ante incentive compatible core in exchange economies with differential information. In this setup, there are no externalities, i.e., only the second difficulty above arises. The basic solution concept in the present paper is an ex ante incentive compatible core for Bayesian games. ${ }^{1}$

Coming back to auctions, we construct a partition form game, which reflects the ex ante commitments of bidding rings. A coalition is core-stable if all its subcoalitions agree to participate in its collusion mechanism. In this definition, we focus on a single ring and assume, as in Marshall and Marx (2007), that the bidders outside the ring do not collude. We first apply the solution concept to standard auctions, without direct externalities. In the case of second price auctions, possibly with asymmetric players, our partition form game reduces to a characteristic function and we prove in proposition 2 that all rings are core-stable. In particular, strategic externalities have limited effects on collusion. Mailath and Zemsky (1991) already obtain this result. They directly focus on the equilibrium in weakly dominant strategies so that they can deal with every coalition separately, without taking account of possible externalities. They thus face a single mechanism design problem for every coalition and derive a characteristic function.

In first price auctions, we derive a genuine partition form game. As is well-known, asymmetric bidders are difficult to handle in this case (see

\footnotetext{
${ }^{1}$ Forges, Minelli and Vohra (2002) discuss interim collective participation constraints in the absence of externalities. The latter assumption takes the form of "orthogonal coalitions" in Myerson (1984)'s study of interim binding agreements in Bayesian games and in Myerson (2007)'s definition of an interim incentive compatible core. A. Kalai and E. Kalai (2009) propose a cooperative-competitive solution to two-person Bayesian games. They consider interim participation but with only two players, incentives only matter for the grand coalition, which does not face externalities.
} 
Krishna (2002)). Lebrun (1991, 1999), Marshall et al. (1994), Waehrer (1999) and Bajari (2001)) introduce bidding rings in first price auctions as prototypes of asymmetric bidders. However, in these papers, rings operate as single entities, which automatically share their information, without relying on any (incentive compatible) mechanism. It follows from our proposition 1 that this simplifying assumption is fully justified if bidding rings can make inside transfers. Thanks to results of Lebrun (1999) and Waehrer (1999), we establish that the grand coalition is always core-stable in a first price auction (proposition 3). In the absence of general, analytical solutions for first price auctions with asymmetric bidders, we only check that all coalitions are corestable in two specific examples, borrowed from McAfee and McMillan (1992) and Marshall et al. (1994).

We finally consider the effect of direct externalities on collusion. We first assume, as in Jehiel and Moldovanu (1996)'s model, that a bidder suffers more if a competitor wins the auction than if the object is not sold at all ("negative externalities"). We check to which extent the grand coalition is (not) core-stable in this case. We then propose a three person first price auction game in which a two bidder ring is not stable. If direct externalities can possibly be positive, we show that the grand coalition is not core-stable and that there exist "small" (i.e., non-singleton) rings which are core-stable. All these examples confirm that direct externalities make cooperative behavior difficult, which was already suggested in Jehiel and Moldovanu (1996), but we give a more precise content to that phenomenon. Indeed, Jehiel and Moldovanu (1996) only show that, under reasonable assumptions, no agreement between (some of) the buyers and/or the seller can be stable. They thus depart from collusion of the bidders in the original auction game.

The paper is organized as follows. Section 2 is devoted to the model and solution concept. In subsection 2.1, we define coalitional equilibria in games with incomplete information. In subsection 2.2, we address the issue of incentives. Proposition 1 and its corollary state that every coalitional equilibrium become incentive compatible once appropriate balanced transfers are made in every coalition. In subsection 2.3, we propose a notion of core-stability for a bidding ring, which does not necessarily gather all the bidders. In section 3 , we apply core-stability to auctions. As a benchmark, we consider standard auctions (second price in subsection 3.1 and first price in subsection 3.2). In subsection 3.3, we turn to auctions with direct externalities. 


\section{Model and solution concept}

\subsection{From Bayesian games to cooperative games}

Let us fix a Bayesian game with independent, private values $\Gamma \equiv\left[N,\left\{T_{i}, q_{i}, A_{i}, u_{i}\right\}_{i \in N}\right]$, namely a set of players $N$ and for every player $i, i \in N$,

- a set of types $T_{i}$

- a probability distribution $q_{i}$ over $T_{i}$

- a set of actions $A_{i}$

- a utility function $u_{i}: T_{i} \times A \rightarrow \mathbb{R}$, where $A=\prod_{i \in N} A_{i}$.

Let $P$ be a coalition structure, namely a partition of $N$. From $\Gamma$ and $P$, we construct an auxiliary Bayesian game $\Gamma(P) \equiv\left[P,\left\{T_{S}, q_{S}, A_{S}, U_{S}\right\}_{S \in P}\right]$, in which the players are the coalitions $S, S \in P$, and

- $T_{S}=\prod_{i \in S} T_{i}, q_{S}=\bigotimes_{i \in S} q_{i}, A_{S}=\prod_{i \in S} A_{i}$

- $U_{S}\left(t_{S},\left(a_{K}\right)_{K \in P}\right)=\sum_{i \in S} u_{i}\left(t_{i},\left(a_{K}\right)_{K \in P}\right)$, where $t_{S}=\left(t_{i}\right)_{i \in S}, a_{K}=$ $\left(a_{i}\right)_{i \in K}$

A strategy ${ }^{2}$ of $S$ in $\Gamma(P)$ is a mapping $\sigma_{S}: T_{S} \rightarrow A_{S}$. Such a definition makes sense if the members of coalition $S$ fully share their information in $T_{S}$ before jointly deciding on an action profile in $A_{S}$. We justify such strategies in the next subsection by showing that they can be derived from coalitions' mechanisms, which allow for appropriate transfers between the coalitions' members. Thanks to these mechanisms, utilities become transferable and incentive compatibility conditions are satisfied (see proposition 1).

As in Ray and Vohra (1997) and Ray (2007), we define a coalitional equilibrium relative to $P$ as a Nash equilibrium $\left(\sigma_{S}\right)_{S \in P}$ of $\Gamma(P)$. We assume that for every $P$, there exists a coalitional equilibrium relative to $P$ and in case of multiple equilibria, we fix a mapping $\sigma$ associating a coalitional

\footnotetext{
${ }^{2}$ In view of our application to auctions, we focus on pure strategies.
} 
equilibrium $\sigma(P)$ with every $P .{ }^{3}$ We denote as $v_{\sigma}(S ; P)$ the expected utility of $S$ at $\sigma(P)$, for every $S \in P$, namely

$$
v_{\sigma}(S ; P)=E\left[\sum_{i \in S} u_{i}\left(\widetilde{t_{i}}, \sigma(P)(\widetilde{t})\right)\right]
$$

where r.v.'s are denoted with $\mathrm{a}^{\sim}$ and $\sigma(P)(\widetilde{t})=\left(\sigma(P)_{K}\left(\widetilde{t}_{K}\right)\right)_{K \in P} .(1)$ defines a partition form game, which is constructed from $\Gamma$ and $\sigma$, with $\Gamma(P)$ as an intermediary step.

Let $T=\prod_{i \in N} T_{i}$. By evaluating (1) at the grand coalition $N$, we get

$$
v_{\sigma}(N ; P) \equiv v(N)=\max _{\tau \in A^{T}} E\left[\sum_{i \in N} u_{i}\left(\widetilde{t}_{i}, \tau(\widetilde{t})\right)\right] \quad \text { for every } \sigma \text { and } P .
$$

$v(N)$ is thus the first best Pareto optimal payoff of the grand coalition. Given any coalitional equilibrium mapping $\sigma$ and any partition $P$ of $N, \sigma(P)$ is a feasible strategy for $N$ (i.e., $\sigma(P) \in A^{T}$ ). Hence, $v_{\sigma}$ is "grand coalition superadditive", or, according to an equivalent terminology, $N$ is efficient in $v_{\sigma}$ :

$$
v(N) \geq \sum_{S \in P} v_{\sigma}(S ; P) \quad \text { for every } P
$$

\subsection{Coalitions' mechanisms}

Let us fix a coalition $S$. A mechanism $\mu_{S}$ for $S$ is a pair of mappings $\mu_{S}=$ $\left(\tau_{S}, m_{S}\right)$ :

$$
\begin{array}{ll}
\tau_{S}: & T_{S} \rightarrow A_{S} \\
m_{S}: & T_{S} \rightarrow\left\{z \in \mathbb{R}^{S}: \sum_{i \in S} z_{i} \leq 0\right\}
\end{array}
$$

\footnotetext{
${ }^{3}$ Ray and Vohra (1997) give sufficient conditions for the existence of a coalitional equilibrium but their result is not useful in our applications to auctions. However, many specific results are available in this context (see section 3). Ray (2007) argues that the partition form game only makes sense if a unique coalitional equilibrium can be associated with every partition (possibly up to transfers). We rather take the view that in case of multiple equilibria, some "standard of behavior" allows us to select among them. Again, this seems appropriate in the context of auctions.
} 
$\tau_{S}$ is $S$ 's decision scheme and $m_{S}$ is a balanced transfer scheme. As usual, the interpretation is that members of $S$ are invited to report their types to a planner who then chooses a profile of actions and transfers as a function of these reports only ${ }^{4}$. According to Marshall and Marx (2007)'s terminology, we use "bid submission mechanisms", in which the bidders' delegate their decision power to a planner (as opposed to "bid coordination mechanisms", in which the planner just recommends bids to the players).

We assume that utilities over mechanisms are quasi-linear. More precisely, the utility of $\mu_{S}$ for player $i \in S$, given his type $t_{i}$, reported types $r_{S}=$ $\left(r_{j}\right)_{j \in S}$, a "strategy" $\sigma_{N \backslash S}: T_{N \backslash S} \rightarrow A_{N \backslash S}$ for the players outside $S$ (e.g., $\sigma_{N \backslash S}=\left(\sigma_{K}\right)_{K \in P, K \neq S}$, for some partition $P$ of $\left.N\right)$ and types $t_{N \backslash S}$ for the players outside $S$ is

$$
u_{i}\left(t_{i}, \tau_{S}\left(r_{S}\right), \sigma_{N \backslash S}\left(t_{N \backslash S}\right)\right)+m_{S}^{i}\left(r_{S}\right)
$$

As this expression explicitly shows, every member $i$ of $S$ incurs an externality from the strategic choices of the players in $N \backslash S$ but, thanks to the private value assumption, does not face any direct informational externality. We define the incentive compatibility (I.C.) of the mechanism $\mu_{S}$ given a mapping $\sigma_{N \backslash S}: T_{N \backslash S} \rightarrow A_{N \backslash S}$. More precisely, $\mu_{S}$ is I.C. given $\sigma_{N \backslash S}$ iff for every $i \in S$, every type $t_{i}$ and reported type $r_{i}$,

$$
\begin{aligned}
& E\left[u_{i}\left(t_{i}, \tau_{S}\left(t_{i}, \widetilde{t}_{S \backslash i}\right), \sigma_{N \backslash S}\left(\widetilde{t}_{N \backslash S}\right)\right)+m_{S}^{i}\left(t_{i}, \widetilde{t}_{S \backslash i}\right)\right] \\
\geq & E\left[u_{i}\left(t_{i}, \tau_{S}\left(r_{i}, \widetilde{t}_{S \backslash i}\right), \sigma_{N \backslash S}\left(\widetilde{t}_{N \backslash S}\right)\right)+m_{S}^{i}\left(r_{i}, \widetilde{t}_{S \backslash i}\right)\right]
\end{aligned}
$$

This definition makes sense because, in any coalitional equilibrium, coalition $S$ must take account of the behavior of the players in $N \backslash S$ in elaborating its own strategy. In the case of complete information, $S$ just looks for a best reply to $N \backslash S$ 's action profile. In the case of incomplete information with private values, $S$ looks for an I.C. best reply to $N \backslash S$ 's strategy $\sigma_{N \backslash S}$, without entering the details of $\sigma_{N \backslash S}$ (whether the players lie or not, how they possibly gather into subcoalitions, etc.). The next proposition justifies the coalitions' strategies in the auxiliary Bayesian game; in particular, we show that explicit I.C. conditions are not necessary. The construction, which goes

\footnotetext{
${ }^{4}$ Proposition 1 below only necessitates interim transfers, which are defined over (the set of reports) $T_{S}$. In particular, we do not rely on transfers depending on the actual players' utilities as in A. Kalai and E. Kalai (2009). In this case, transfers also depend on the players' types.
} 
back to Arrow (1979) and d'Aspremont and Gérard-Varet (1979, 1982), has been widely used in economic frameworks which do not involve externalities (see, e.g., Forges et al. (2002)).

Proposition 1 Let $S \subseteq N$; let $\sigma_{N \backslash S}: T_{N \backslash S} \rightarrow A_{N \backslash S}$ be an arbitrary strategy of $N \backslash S$ and let $\sigma_{S}$ be a best response of $S$ to $\sigma_{N \backslash S}$ in $\Gamma(\{S, N \backslash S\})$. There exists a transfer scheme $m_{S}$ such that

1. $\sum_{i \in S} m_{S}^{i}\left(r_{S}\right)=0$ for every $r_{S} \in T_{S}$

2. The mechanism $\left(\sigma_{S}, m_{S}\right)$ is I.C. given $\sigma_{N \backslash S}$.

Proof: Let us fix $S, \sigma_{N \backslash S}$ and $\sigma_{S}$ as in the statement. For every $i \in S, t_{i} \in$ $T_{i}, a_{S} \in A_{S}$ let us set

$$
h_{i}\left(t_{i}, a_{S}\right)=E\left[u_{i}\left(t_{i}, a_{S}, \sigma_{N \backslash S}\left(\widetilde{t}_{N \backslash S}\right)\right)\right]
$$

Since $\sigma_{S}$ is a best response to $\sigma_{N \backslash S}$,

$$
\sum_{i \in S} h_{i}\left(t_{i}, \sigma_{S}\left(t_{S}\right)\right) \geq \sum_{i \in S} h_{i}\left(t_{i}, a_{S}\right) \quad \forall t_{S} \in T_{S}, a_{S} \in A_{S}
$$

Let $\widehat{m}_{S}^{i}\left(r_{S}\right)=\sum_{j \in S \backslash i} h_{j}\left(r_{j}, \sigma_{S}\left(r_{S}\right)\right)$. For every $i \in S$, type $t_{i} \in T_{i}$, reported type $r_{i} \in T_{i}$ and reported types $r_{S \backslash i} \in \prod_{j \in S \backslash i} T_{j}$ of the other members of $S$,

$$
\begin{aligned}
h_{i}\left(t_{i}, \sigma_{S}\left(r_{S}\right)\right)+\widehat{m}_{S}^{i}\left(r_{S}\right) & =h_{i}\left(t_{i}, \sigma_{S}\left(r_{S}\right)\right)+\sum_{j \in S \backslash i} h_{j}\left(r_{j}, \sigma_{S}\left(r_{S}\right)\right) \\
& \leq h_{i}\left(t_{i}, \sigma_{S}\left(t_{i}, r_{S \backslash i}\right)\right)+\sum_{j \in S \backslash i} h_{j}\left(r_{j}, \sigma_{S}\left(t_{i}, r_{S \backslash i}\right)\right) \\
& =h_{i}\left(t_{i}, \sigma_{S}\left(t_{i}, r_{S \backslash i}\right)\right)+\widehat{m}_{S}^{i}\left(t_{i}, r_{S \backslash i}\right)
\end{aligned}
$$

where the inequality is due to (4) w.r.t. the type vector $\left(t_{i}, r_{S \backslash i}\right)$.

Hence, the mechanism $\left(\sigma_{S}, \widehat{m}_{S}\right)$ is I.C. given $\sigma_{N \backslash S}$, but not yet balanced. Let $\bar{m}_{S}^{i}\left(r_{i}\right)=E\left[\widehat{m}_{S}^{i}\left(r_{i}, \widetilde{t}_{S \backslash i}\right)\right]$. By taking expectations in (5) we conclude that $\left(\sigma_{S}, \bar{m}_{S}\right)$ is I.C. given $\sigma_{N \backslash S}$. 
Finally, let $m_{S}^{i}\left(r_{S}\right)=\bar{m}_{S}^{i}\left(r_{i}\right)-\frac{1}{|S|-1} \sum_{j \in S \backslash i} \bar{m}_{S}^{j}\left(r_{j}\right)$. Then $\left(\sigma_{S}, m_{S}\right)$ is I.C. given $\sigma_{N \backslash S}$ and $\sum_{i \in S} m_{S}^{i}\left(r_{S}\right)=0$ for every $r_{S} \in T_{S}$.

As a direct consequence of this proposition, we get the following

Corollary Every coalitional equilibrium can be made incentive compatible: let $P$ be a partition of $N$ and $\sigma$ be a coalitional equilibrium relative to $P$; for every $S \in P$, there exists a transfer scheme $m_{S}$ such that $\left(\sigma_{S}, m_{S}\right)$ is I.C. given $\left(\sigma_{K}\right)_{K \in P, K \neq S}$ and

$$
v_{\sigma}(S ; P)=E\left[\sum_{i \in S}\left(u_{i}\left(\widetilde{t}_{i}, \sigma_{S}\left(\widetilde{t}_{S}\right),\left(\sigma_{K}\left(\widetilde{t}_{K}\right)\right)_{K \in P, K \neq S}\right)+m_{S}^{i}\left(\widetilde{t}_{S}\right)\right)\right]
$$

\subsection{Core-stability of a (single) ring}

Let us denote as $\mathcal{P}(K)$ the set of all partitions of $K$, for $K \subseteq N$. Let $R \subseteq N$; from $v_{\sigma}(S ; P)$, we derive the following characteristic function over $R$

$$
w_{\sigma}^{R}(S)=\min _{\Pi \in \mathcal{P}(R \backslash S)} v_{\sigma}\left(S ;\left\{S, \Pi,\{k\}_{k \in N \backslash R}\right\}\right)
$$

In particular, for the grand coalition $N$,

$$
w_{\sigma}^{N}(S)=\min _{\Pi \in \mathcal{P}(N \backslash S)} v_{\sigma}(S ;\{S, \Pi\})
$$

We say that $R$ is core-stable (w.r.t. $\sigma$ ) iff the (standard) core of $w_{\sigma}^{R}, C\left(w_{\sigma}^{R}\right)$, is not empty. The interpretation is the following:

- The coalitional equilibrium mapping $\sigma$ is given.

- The ring $R$ considers to form; the players outside $R$ are supposed to act individually. $R$ proposes to every $i \in R$ a share $x_{i}$ of the total expected payoff $w_{\sigma}^{R}(R)=v_{\sigma}\left(R ;\left\{R,\{k\}_{k \in N \backslash R}\right\}\right)$, to be achieved by means of an I.C. mechanism $\mu_{R}=\left(\sigma_{R}, m_{R}\right)$. 
- Every subcoalition $S$ of $R$ considers non-participation; if $S$ does not participate, the players outside $R$ remain singletons, the players in $R \backslash S$ partition themselves as they wish. Hence $S$ can guarantee the total expected payoff $w_{\sigma}^{R}(S)$ to its members.

- If the participation constraint of every $S \subseteq R$ is satisfied, $R$ forms; every player observes his type; $R$ implements $\mu_{R}$.

\section{Basic properties}

- Every singleton $\{k\}, k \in N$, is core-stable.

- Recalling (2), for every $\sigma, w_{\sigma}^{N}(N)=v(N)$; by (3) and (6), $w_{\sigma}^{N}$ is grand coalition superadditive $\left(N\right.$ is efficient in $\left.w_{\sigma}^{N}\right)$. This property does not necessarily hold for $w_{\sigma}^{R}, R \varsubsetneqq N$ (see example 2 in section 3.3).

- $C\left(w_{\sigma}^{R}\right)$ corresponds to cautious expectations of the subcoalitions of $R$. In particular, $C\left(w_{\sigma}^{N}\right)$ contains the usual variants of the core of the partition form game $v_{\sigma}$ (see Hafalir (2007)). For instance, the core with singleton expectations, or $s$-core, of $v_{\sigma}$, denoted as $C_{s}\left(v_{\sigma}\right)$, is defined as the standard core $C\left(f_{\sigma}^{s}\right)$ of the characteristic function

$$
f_{\sigma}^{s}(S)=v_{\sigma}\left(S ;\left\{S,\{j\}_{j \in N \backslash S}\right\}\right)
$$

Similarly, the core with merging expectations (see Maskin (2003)), or $m$-core, of $v_{\sigma}, C_{m}\left(v_{\sigma}\right)$, is defined as $C_{m}\left(v_{\sigma}\right)=C\left(f_{\sigma}^{m}\right)$, where

$$
f_{\sigma}^{m}(S)=v_{\sigma}(S ;\{S, N \backslash S\})
$$

It readily follows from the definitions that $C\left(f_{\sigma}^{s}\right)$ and $C\left(f_{\sigma}^{m}\right)$ are subsets of $C\left(w_{\sigma}^{N}\right)$. Unlike $w_{\sigma}^{N}$, the characteristic functions $f_{\sigma}^{s}$ and $f_{\sigma}^{m}$ are not necessarily grand coalition superadditive (see example 1 in section 3.3).

- Equivalent definition: $w_{\sigma}^{N}$ can be defined in terms of the conjecture of every coalition $S$ on the partition to be formed by the players of $N \backslash S$ if $S$ secedes from the grand coalition $N$. For every coalition $S$, let $B(S)$ be a partition of $N$ which contains $S$ as a cell. Given a partition form game $v$, let $f^{B}(S)=v(S ; B(S))$. The $B$-core of $v$ is defined as the core of the characteristic function game $f^{B}$. The $s$-core and the 
$m$-core correspond respectively to $B(S)=\{S ;\{j\}, j \in N \backslash S\}$ and $B(S)=\{S, N \backslash S\}$ for every $S$. The grand coalition $N$ is then corestable (w.r.t. $\sigma$ ) if, for some specification of the conjecture $B(S)$ of every coalition $S$, the $B$-core of $v_{\sigma}$ is not empty.

- If $\Gamma$ is a game with complete information, let

$$
v_{\alpha}(S)=\max _{a_{S} \in A_{S}} \min _{a_{N \backslash S} \in A_{N \backslash S}}\left[\sum_{i \in S} u_{i}\left(a_{S}, a_{N \backslash S}\right)\right]
$$

In particular, $v_{\alpha}(N)=v(N)$. The $\alpha$-core of $\Gamma$ is defined as $C\left(v_{\alpha}\right)$ (see Aumann (1961)). It is easily checked that, for every $\sigma$ and every $S \varsubsetneqq N$, $w_{\sigma}^{N}(S) \geq v_{\alpha}(S)$. Hence, $C\left(w_{\sigma}^{N}\right) \subseteq C\left(v_{\alpha}\right) .^{5}$ The extension of the definition of the $\alpha$-core to incomplete information may be delicate in the presence of incentive constraints. In particular, our previous construction of transfers, which made any coalitional equilibrium incentive compatible (see proposition 1), cannot be used for the maxmin, since the latter solution concept requires that coalition $S$ considers any possible strategy of coalition $N \backslash S{ }^{6}$ However, in the framework of standard auctions, the difficulties disappear. Indeed, every coalition $S$ guarantees itself a total expected payoff of 0 , whatever the mechanism adopted by $N \backslash S$, by having all its members bidding 0 independently of their types, a strategy that is clearly I.C. for $S$. Furthermore, $S$ cannot guarantee more than 0 , since the members of $N \backslash S$ can all bid the maximal possible amount, which is I.C. for $N \backslash S$. Hence, the $\alpha$-core is well-defined and not empty in standard auctions. But the usual objection against maxmin strategies applies: why should $S$ fear costly overbidding from $N \backslash S ?$

\footnotetext{
${ }^{5}$ Hafalir (2007) focuses on abstract partition form games, which are not necessarily generated by a strategic form game. Hence he does not distinguish the core with cautious expectations from the $\alpha$-core. In our framework, at least under complete information, Aumann (1961)'s original definition of the $\alpha$-core can be used.

${ }^{6}$ Our construction applies to the minmax, i.e., to the $\beta$-characteristic function, in the sense that we can dispense with I.C. constraints in the best replies of the coaltion under consideration.
} 


\section{Applications}

In this section, we apply our solution concept to auctions with independent private values. In the first two subsections, we consider standard auctions, that is, without direct externalities. We check the core-stability of coalitions in several specific auction models which have been proposed in the literature. In subsections 3.1 and 3.2, we illustrate that, in absence of direct externalities, coalitions are core-stable. In subsection 3.3, we allow for direct negative externalities. We show that the grand coalition can be made core-stable in this case. However, the $s$-core and the $m$-core of the underlying partition form game can be empty (example 1) and small coalitions may not be corestable (example 2). Finally, if externalities are possibly positive, the $\alpha$-core may be empty (example 3).

\subsection{Standard second price auctions}

Let player $i$ 's type $\widetilde{t}_{i}$ be a continuous random variable over $\left[\underline{t}_{i}, \bar{t}_{i}\right], 0 \leq \underline{t}_{i} \leq \bar{t}_{i}$, to be interpreted as his valuation for a single object. $A_{i}=[0, M]$ is the set of possible bids, where $M \geq \max _{i \in N} \bar{t}_{i}$. Let $a=\left(a_{k}\right)_{k \in N}$ be an $n$-tuple of bids. A second price auction is defined by the following utility functions

$$
\begin{aligned}
u_{i}\left(t_{i}, a\right) & =t_{i}-\max _{j \neq i} a_{j} \quad \text { if } a_{i}>\max _{j \neq i} a_{j} \\
& =\frac{1}{\eta(a)}\left(t_{i}-a_{i}\right) \quad \text { if } a_{i}=\max _{j \neq i} a_{j} \\
& =0 \text { otherwise }
\end{aligned}
$$

where $\eta(a)=\left|\left\{k \in N: a_{k}=\max _{j \in N} a_{j}\right\}\right|$.

As is well-known, this game has an equilibrium in weakly dominant strategies. More generally, let $P$ be a partition of $N$. The auxiliary Bayesian game $\Gamma(P)$ has a coalitional equilibrium in weakly dominant strategies described by $\sigma_{S}^{k}\left(t_{S}\right)=t_{k}$ for some $k \in S$ such that $t_{k}=\max _{j \in S} t_{j}$ and $\sigma_{S}^{i}\left(t_{S}\right)=0$ for $i \in S, i \neq k$, for every $S \in P$ and $t_{S}=\left(t_{j}\right)_{j \in S}$. It is easily checked that for every $P$ and $S \in P$,

$$
v_{\sigma}(S ; P)=v_{\sigma}(S ;\{S, N \backslash S\})=E\left[\left(\max _{i \in S} \widetilde{t}_{i}-\max _{j \in N \backslash S} \widetilde{t}_{j}\right)^{+}\right] \equiv \varphi(S)
$$


where $f^{+}=\max \{f, 0\}$. The previous expression shows that, at the equilibrium in weakly dominant strategies, the external effects disappear, so that $v_{\sigma}$ reduces to a plain characteristic function. In particular, for every $S \subseteq R \subseteq N$ and every $\Pi \in \mathcal{P}(R \backslash S), v_{\sigma}\left(S ;\left\{S, \Pi,\{k\}_{k \in N \backslash R}\right\}\right)=\varphi(S)$ and a ring $R$ is core-stable iff $C\left(\left.\varphi\right|_{R}\right)$ is not empty, where $\left.\varphi\right|_{R}(S)=\varphi(S)$ for every $S \subseteq R$.

Proposition 2 (Mailath and Zemsky (1991), Barbar and Forges (2007)) In a standard second price auction, all rings are core-stable.

Proof: Mailath and Zemsky (1991) establish that $\varphi$ is balanced. Barbar and Forges (2007) further show that $\varphi$ is supermodular (convex). If the bidders are symmetric, namely if the types $\widetilde{t}_{i}, i=1, \ldots, n$, are i.i.d., an easy direct argument shows that giving the same amount $\frac{\varphi(N)}{|N|}$ to every member of $N$ defines a payoff $n$-tuple in $C(\varphi)$ : first, $I$ denoting the indicator function,

$$
\varphi(S) \leq E\left[\left(\max _{i \in S} \widetilde{t}_{i}\right) I\left[\max _{i \in S} \widetilde{t}_{i} \geq \max _{j \in N \backslash S} \widetilde{t}_{j}\right]\right]
$$

Further, it is easily checked that

$$
\begin{aligned}
& P\left(\left\{\max _{i \in S} \tilde{t}_{i} \leq t\right\} \cap\left\{\max _{i \in S} \tilde{t}_{i} \geq \max _{j \in N \backslash S} \tilde{t}_{j}\right\}\right) \\
= & P\left(\left\{\max _{i \in N} \widetilde{t}_{i} \leq t\right\} \cap\left\{\max _{i \in S} \widetilde{t}_{i} \geq \max _{j \in N \backslash S} \tilde{t}_{j}\right\}\right) \\
= & F^{n}(t) \frac{|S|}{|N|}
\end{aligned}
$$

where $F$ is the distribution function of any $\widetilde{t}_{i}$. It follows then from (10) that $\varphi(S) \leq \varphi(N) \frac{|S|}{|N|}$.

\subsection{Standard first price auctions}

In this subsection, we assume that the $n$ initial bidders are symmetric, namely that the valuations $\widetilde{t}_{i}, i=1, \ldots, n$ are i.i.d. Let $a=\left(a_{k}\right)_{k \in N}$ be an $n$-tuple of bids. A first price auction is defined by the following utility functions

$$
\begin{aligned}
u_{i}\left(t_{i}, a\right) & =t_{i}-a_{i} \quad \text { if } a_{i}>\max _{j \neq i} a_{j} \\
& =\frac{1}{\eta(a)}\left(t_{i}-a_{i}\right) \quad \text { if } a_{i}=\max _{j \neq i} a_{j} \\
& =0 \text { otherwise }
\end{aligned}
$$


where $\eta(a)$ is defined as for the second price auction.

Obviously, given a nontrivial partition $P$ of $N$, the players of the auxiliary Bayesian game $\Gamma(P)$ are not symmetric. By Lebrun $(1999), \Gamma(P)$ has a unique equilibrium, for every partition $P$. In other words, there exists a unique coalitional equilibrium mapping $\sigma$. However, no general analytical solution is available.

Waehrer (1999, proposition 2) shows that for every partition $P$ and every coalitions $R, S \in P$ such that $|R| \leq|S|$

$$
\frac{v_{\sigma}(S ; P)}{|S|} \leq \frac{v_{\sigma}(R ; P)}{|R|}
$$

In words, at a first price auction, the per capita expected payoff of a cartel's member is greater in small cartels ${ }^{7}$. This result enables us to deduce the following

Proposition 3 In a standard first price auction with symmetric bidders, the grand coalition is core-stable.

Proof: We will show that the vector payoff allocating the amount $\frac{v(N)}{|N|}$ to every member of $N$ is in the $s$-core of the underlying partition game $v_{\sigma}$. Let $S \varsubsetneqq N$ and $P=\left\{S,\{k\}_{k \in N \backslash S}\right\}$. Recalling the definition of the $s$-core (see (7)), we have to show that

$$
\frac{v(N)}{|N|} \geq \frac{v_{\sigma}(S ; P)}{|S|}
$$

From (11), we deduce that for every $j \in N \backslash S$,

$$
v_{\sigma}(\{j\} ; P) \geq \frac{v_{\sigma}(S ; P)}{|S|}
$$

while, from the grand coalition superadditivity of $v_{\sigma}$ (recall (3)),

$$
v(N) \geq v_{\sigma}(S ; P)+\sum_{j \in N \backslash S} v_{\sigma}(\{j\} ; P)
$$

The latter two inequalities yield (12).

\footnotetext{
${ }^{7}$ Waehrer (1999) also shows that for second price auctions, the inequality goes the other way round.
} 
The previous reasoning can be applied to establish the stability of a bidding ring $R \varsubsetneqq N$ if $v_{\sigma}$ is superadditive on $R$. Such a property indeed holds in examples proposed by McAfee and McMillan (1992) and Marshall et al. (1994).

McAfee and McMillan (1992) assume that $\widetilde{t}_{i} \in\{0,1\}, i=1, \ldots, n$. They show (in inequality (13)) that, for every coalition $S \varsubsetneqq N$ and $j \in N \backslash S$,

$$
\begin{aligned}
& v_{\sigma}\left(S \cup\{j\} ;\left\{S \cup\{j\},\{k\}_{k \in N \backslash(S \cup\{j\})}\right\}\right) \\
\geq & v_{\sigma}\left(S ;\left\{S,\{k\}_{k \in N \backslash S}\right\}\right)+v_{\sigma}\left(\{j\} ;\left\{S,\{k\}_{k \in N \backslash S}\right\}\right)
\end{aligned}
$$

or, equivalently, recalling our notation $f_{\sigma}^{s}$ (see $(7)$ )

$$
f_{\sigma}^{s}(S \cup\{j\}) \geq f_{\sigma}^{s}(S)+v_{\sigma}\left(\{j\} ;\left\{S,\{k\}_{k \in N \backslash S}\right\}\right)
$$

One can also check that (11) holds in their framework so that

$$
v_{\sigma}\left(\{j\} ;\left\{S,\{k\}_{k \in N \backslash S}\right\}\right) \geq \frac{f_{\sigma}^{s}(S)}{|S|}
$$

Hence

$$
\frac{f_{\sigma}^{s}(S \cup\{j\})}{|S|+1} \geq \frac{f_{\sigma}^{s}(S)}{|S|}
$$

and, by induction, for every coalitions $R, S$ such that $S \subseteq R$,

$$
\frac{f_{\sigma}^{s}(R)}{|R|} \geq \frac{f_{\sigma}^{s}(S)}{|S|}
$$

Since $f_{\sigma}^{s}(S) \geq w_{\sigma}^{R}(S)$ for $S \subseteq R$, with equality if $S=R$, the latter inequality implies that every ring $R$ is core-stable in McAfee and McMillan (1992)'s example.

Marshall et al. (1994) compute $f_{\sigma}^{s}$ by numerical methods in the case of five initial bidders uniformly distributed over $[0,1]$. Their table III shows that $\frac{f_{\sigma}^{s}(S)}{|S|}$ is increasing with the size of $S$ (i.e., (13) holds) so that, in their example too, all rings are core-stable.

\subsection{First price auction with complete information and direct externalities}

Following Jehiel and Moldovanu (1996), we consider first price auctions with complete information, in which every bidder incurs an externality if a competitor acquires the object. The basic game reduces to $\Gamma \equiv\left[N,\left\{A_{i}, u_{i}\right\}_{i \in N}\right]$, 
where $A_{i}=\{0, \epsilon, 2 \epsilon, \ldots\}$ is the set of possible bids, given a smallest money unit $\epsilon>0$. The utility functions are described by an $n \times n$ matrix $E=\left[e_{i j}\right]$; for every $i, e_{i i} \equiv t_{i}$ is agent $i$ 's utility for the object and for every $i \neq j, e_{i j}$ is the externality incurred by agent $j$ if agent $i$ gets the object. If all bids are 0 , the seller keeps the object; agent $i$ 's utility is normalized to 0 in this case. Let $a=\left(a_{k}\right)_{k \in N}$; the utility of player $i$ is

$$
\begin{aligned}
u_{i}(a) & =t_{i}-a_{i} \quad \text { if } a_{i}>\left[\max _{j \neq i} a_{j}\right]^{+} \\
& =e_{j i} \quad \text { if } a_{j}>\left[\max _{k \neq j} a_{k}\right]^{+} \text {for some } j \neq i \\
& =0 \text { if } a=0
\end{aligned}
$$

To complete this description, we assume that if several players make the highest bid, they all get the object with the same probability.

Recalling (2), we have here

$$
v(N)=\max _{a \in A}\left[\sum_{i \in N} u_{i}(a)\right]=\left[\max _{i \in N}\left\{t_{i}+\sum_{j \neq i} e_{i j}\right\}-\epsilon\right]^{+}
$$

Since $\Gamma$ is a game with complete information, the $\alpha$-characteristic function $v_{\alpha}$ is defined by $(9)$.

\section{Core-stability of the grand coalition under negative externalities}

Except in example 3, we assume negative externalities, i.e., $e_{i j} \leq 0$ for every $i \neq j$. In this case, given any strategy profile $\left(a_{i}\right)_{i \in S}$ of $S, N \backslash S$ can inflict a negative payoff on $S$ by bidding over $\max _{i \in S} a_{i}$; hence $v_{\alpha}(S) \leq 0$ for $S \varsubsetneqq N$; since $v(N) \geq 0$, the $\alpha$-core $C\left(v_{\alpha}\right)$ is not empty. A similar argument shows that, for a coalitional equilibrium mapping $\sigma$ proposed in Jehiel and Moldovanu $(1996)^{8}, w_{\sigma}^{N}(N)=v(N) \geq 0$, while for every $S \varsubsetneqq N, w_{\sigma}^{N}(S) \leq 0$.

\footnotetext{
${ }^{8}$ Jehiel and Moldovanu (1996) prove that, under appropriate genericity conditions, the following strategies $\left(b_{j}\right)_{j \in N}$ form an equilibrium in $\Gamma$ : if $t_{i}-\min _{j} e_{j i} \leq 0$ for every $i=1, \ldots, n$, then $b_{i}=0$ for every $i$. Otherwise, let $(i, k)$ be a pair of bidders $i \neq k$ such that $t_{i}-e_{k i}$ is maximal over all $t_{j}-e_{l j}, j \neq l$ (that is, bidder $i$ is willing to pay the highest price for the object, given his valuation and the externalities he might suffer); take $b_{i}=t_{i}-e_{k i}-\epsilon, b_{k}=t_{i}-e_{k i}-2 \epsilon$ and $b_{j}<b_{k}, j \neq i, k$. At this equilibrium, which typically involves weakly dominated strategies, bidder $i$ 's payoff is $e_{k i}+\epsilon \leq 0$ and all other bidders $j \neq i$ get $e_{i j} \leq 0$ (see Biran (2009), Appendix A, for a full characterization of equilibria).
} 
Hence, for that particular choice of $\sigma$, the grand coalition $N$ is core-stable, namely $C\left(w_{\sigma}^{N}\right) \neq \emptyset$. Jehiel and Moldovanu (1996) establish the emptiness of the $\alpha$-core of a quite different market game, in which all agreements between the bidders and the seller are possible. Here, we stick to the original format of the first price auction, so that we only allow for collusion between the potential buyers.

At the above coalitional equilibrium mapping $\sigma$, all conceivable cores (e.g., the $s$-core and the $m$-core, see subsection 2.3 ) are nonempty. The example below illustrates that this property does not necessarily hold for coalitional equilibrium mappings which lead to possibly positive payoffs. ${ }^{9}$

Example 1: $n=4$; the matrix of valuations/externalities is

$$
E=\left(\begin{array}{cccc}
t_{1} & -2 & -2 & -2 \\
0 & 1 & 0 & 0 \\
-8 & -8 & 1 & -8 \\
-7 & -7 & -7 & 1
\end{array}\right)
$$

Let us start with $t_{1}=8 \cdot v(N)=2-\epsilon$. Assume first that the bidders act individually. Then the following strategies form an equilibrium: $a_{1}=a_{4}=$ $8-2 \epsilon, a_{2}=8, a_{3}=8-\epsilon$. Bidder 2 wins the auction and the payoffs are $(0,-7,0,0)$. Hence,

$$
v_{\sigma}(\{i\} ;\{\{1\},\{2\},\{3\},\{4\}\})=0, i=3,4
$$

Assume next that the first two bidders collude, while the two others remain singletons. The relevant matrix becomes

$$
\left(\begin{array}{ccc}
6 & -2 & -2 \\
-16 & 1 & -8 \\
-14 & -7 & 1
\end{array}\right)
$$

The following strategies now form an equilibrium: $a_{1}=3, a_{2}=0, a_{3}=$ $3-\epsilon, a_{4}=3-2 \epsilon$. Coalition $\{1,2\}$ gets a payoff of 3 so that

$$
v_{\sigma}(\{1,2\} ;\{\{1,2\},\{3\},\{4\}\})=3
$$

\footnotetext{
${ }^{9}$ The features of the next examples depend crucially on the direct externalities. In a first price auction with complete information and no externalities, there exists a coalitional equilibrium mapping $\sigma$ in which the outcome (namely, the winner and the price) is as in the equilibrium in undominated strategies of the second price auction. For that $\sigma$, the $s$-core and the $m$-core of $v_{\sigma}$ are not empty. Furthermore, every bidding ring is core-stable w.r.t. $\sigma$.
} 
(14) and (15) imply that the characteristic function $f_{\sigma}^{s}$ is not grand coalition superadditive, hence that the $s$-core $C_{s}\left(v_{\sigma}\right)$ is empty in that example.

Let us take $t_{1}=4$. We now have $v(N)=1-\epsilon$. Let us assume that bidder 3 competes with the cartel $\{1,2,4\}$. The matrix is

$$
\left(\begin{array}{cc}
1 & 0 \\
-24 & 1
\end{array}\right)
$$

where the first row corresponds to the utilities in case the cartel obtains the object. The strategies $a_{1}=0, a_{2}=1, a_{3}=1-\epsilon, a_{4}=0$ form an equilibrium. Hence

$$
v_{\sigma}(\{3\} ;\{\{3\},\{1,2,4\}\})=0
$$

and similarly for bidder 4 . Let us assume again that the first two bidders collude, but facing the opposite ring $\{3,4\}$. The relevant matrix is now

$$
\left(\begin{array}{cc}
2 & -4 \\
-14 & -6
\end{array}\right)
$$

The strategies $a_{1}=\epsilon, a_{2}=a_{3}=a_{4}=0$ are in equilibrium, so that

$$
v_{\sigma}(\{1,2\} ;\{\{1,2\},\{3,4\}\})=2-\epsilon
$$

(16), the analog of (16) for bidder 4 and (17) imply that the characteristic function $f_{\sigma}^{m}$ is not grand coalition superadditive, hence that the $m$-core $C_{m}\left(v_{\sigma}\right)$ is empty in that example.

Core-stability of a "small" coalition under negative externalities

Jehiel and Moldovanu (1996) relate the case of two European firms who did not cooperate in a procurement auction opposing them to an Asian competitor. They suggest that negative externalities might explain the failure of the natural partners' association but, as explained above, the emptiness of the $\alpha$-core that they consider only shows that no stable agreement can be found between the three potential buyers and the seller. In this particular example, cooperation between the European firms and the Asian one looked unlikely, but the stability of the European coalition could be considered. We illustrate below that, in the presence of externalities, a two firm cartel may not be stable.

Example 2: $n=3$; the matrix of valuations/externalities is 


$$
E=\left(\begin{array}{rrr}
5 & -4 & -3 \\
-4 & 6 & -9 \\
-10 & -1 & 3
\end{array}\right)
$$

If a first price auction takes place between the 3 agents, in every equilibrium, agent 1 wins and agent 3 is the second highest bidder; in undominated strategies, $10 \leq p \leq 12$; at the lowest price $p=10$, the utilities are $(-5,-4,-3)$. Provided that $p<11$, bidders 1 and 2 get a total utility $>-10$. If they form a joint venture, in every equilibrium, agent 2 represents $R=\{1,2\}$ at the auction and wins; in undominated strategies, $p=12$ : the price raises when agent 1 and agent 2 do not compete. The total utility of $\{1,2\}$ is -10 , which is less than the sum of agents 1 and 2's individual payoffs (in our previous notation, $w_{\sigma}^{R}(\{1\})=-5, w_{\sigma}^{R}(\{2\})=-4, w_{\sigma}^{R}(\{1,2\})=-10$ ). The interpretation is the following: if agents 1 and 2 get together, they cannot expect more than -10 ; if agent 3 plays a dominated strategy, they will even get less. If agent 1 breaks the agreement, he does not expect that agents 2 and 3 (like a European firm and the Asian firm above) will collude, but considers a noncooperative equilibrium between the three competitors. At an equilibrium leading to the lowest price, he can expect -5 . Similarly, agent 2 can expect -4 .

Core-stability of the grand coalition under possibly positive externalities

In example 2, the grand coalition is core-stable. If externalities are negative, the grand coalition can decide not to participate in the auction so as to guarantee 0 to its members, a strategy that is not feasible for small coalitions. More generally, the next proposition, proved in the appendix, states that, if $n \leq 3$, the grand coalition is core-stable w.r.t. every coalitional equilibrium mapping, even if externalities can be positive. Recall that $f^{+}=\max \{f, 0\}$.

Proposition 4 In every 3-player first price auction with direct externalities such that $t_{i}>e_{j i}^{+}$for every $i, j \neq i$, the grand coalition is core-stable w.r.t. every coalitional mapping $\sigma$.

We conclude this section by illustrating that, if sufficiently many players face possibly positive externalities, the grand coalition may not be stable. In the next example, with five players, the $\alpha$-core, $C\left(v_{\alpha}\right)$, is empty.

Example 3: $n=5$; every agent $i$ has two neighbors $(i-1 \bmod 5, i+1 \bmod 5)$; $t_{i}=3, e_{j i}=2$ if agent $j$ is a neighbor of agent $i, e_{j i}=-2$ otherwise. 
One computes that $v(N)=3-\epsilon$. By symmetry, if $C\left(v_{\alpha}\right) \neq \emptyset$, the payoff vector in which every agent gets $\frac{3-\epsilon}{5}$ must be in $C\left(v_{\alpha}\right)$. Let us consider a coalition of the form $S=\{i, i+1, i+3\}$ where + is mod 5 , i.e., $S$ contains agent $i$, a neighbor of agent $i$ and a non-neighbor of agent $i$. $S$ guarantees $\max \{3-\epsilon, 2\}$ if agent $i$ bids $\epsilon$ and the other members of $S$ bid 0; hence, $v_{\alpha}(S) \geq 2 \geq 3 \times \frac{3-\epsilon}{5}$, contradicting $C\left(v_{\alpha}\right) \neq \emptyset .{ }^{10}$ Hence the grand coalition is not stable in this example. It can be shown that the same holds for all coalitions of 4 players but that all coalitions of 2 or 3 players are stable, for any coalitional equilibrium mapping in undominated strategies.

\section{Concluding remarks}

In this paper, we study collusion in auctions, possibly with direct externalities, by associating a cooperative game to the initial Bayesian game modelling the auction. Such a simple "semi-cooperative" approach, which constructs a direct "bridge" between the initial noncooperative game and a cooperative one, abstracts from the details of the strategic negotiation between coalitions (see Ray (2007) and A. Kalai and E. Kalai (2009) for recent discussions of this issue). The partition form game $v_{\sigma}(S ; P)$ constructed in this paper is tractable and enables us to use a well-founded solution concept as the core. The auxiliary game Bayesian game behind $v_{\sigma}(S ; P)$ was considered in Marshall et al. (1994) and Waehrer (1999) without any justification for the absence of incentive compatibility constraints. The corollary of proposition 1 provides such a justification.

We give a precise content to the idea that "direct externalities make collusion harder". According to the available results, without direct externalities, bidding rings are stable. Examples based on Jehiel and Moldovanu (1996), i.e., with complete information, show that this property no longer holds in the presence of direct externalities. A natural setup to pursue the analysis is the second price auction with externalities proposed by Caillaud and Jehiel (1998), in which the valuations of the initial bidders are independently and identically distributed. They show that if interim individual participation constraints are imposed, the grand coalition may fail from being ex post efficient but do not address the question of its ex ante stability.

\footnotetext{
${ }^{10}$ Equivalently: $\mathcal{S}=\{\{i, i+1, i+3\}, i=1, \ldots, 5\}$ is balanced (with weights $\lambda_{S}=\frac{1}{3}$ ) and $\sum_{S \in \mathcal{S}} \lambda_{S} v_{\alpha}(S) \geq \frac{10}{3}>3-\epsilon$.
} 
In this paper, we focused on independent private values. This assumption, which is standard in the auction framework, plays a crucial role in dispensing with explicit incentive compatibility constraints in the definition of coalitional equilibria (i.e., in proposition 1). In more general models, we expect that "second best" coalitional equilibria will need to be considered. Our definition of incentive compatibility can be extended to cover general utility functions but the study of coalitional equilibria with I.C. binding constraints is potentially complex.

\section{A Appendix: proof of proposition 4}

Let us fix an arbitrary coalitional mapping $\sigma$, namely, for every partition $P$ of $N=\{1,2,3\}$, a Nash equilibrium $\sigma(P)$ of the auction game in which the players are the coalitions in $P$. We will show that the core with singleton expectations $C_{s}\left(v_{\sigma}\right)$ is not empty, i.e., that $C\left(f_{\sigma}^{s}\right) \neq \emptyset$, where the characteristic function $f_{\sigma}^{s}$ is defined by $(7)$.

Let us assume w.l.o.g. that player 1 is efficient in $N$, namely that

$$
t_{1}+e_{12}+e_{13} \geq \max \left\{t_{2}+e_{21}+e_{23}, t_{3}+e_{31}+e_{32}\right\}
$$

Then

$$
f_{\sigma}^{s}(N)=\left[t_{1}+e_{12}+e_{13}-\epsilon\right]^{+}
$$

We will consider the modified characteristic function $g_{\sigma}$ defined by

$$
\begin{aligned}
g_{\sigma}(N) & =t_{1}+e_{12}+e_{13}-\epsilon \\
g_{\sigma}(S) & =f_{\sigma}^{s}(S) \text { for every } S \varsubsetneqq N
\end{aligned}
$$

and show that $C\left(g_{\sigma}\right) \neq \emptyset$. Let us set $x_{i}=g_{\sigma}(\{i\}), i=1,2,3 . x_{i}$ is player $i$ 's payoff at the equilibrium $\tau \equiv \sigma(\{\{1\},\{2\},\{3\}\})$ induced by $\sigma$ in the 3 -person original auction game. Since $t_{i}>0$ for every $i$, the seller cannot keep the object at $\tau$. If player $i$ gets the object at a positive price $p$ at $\tau$, $x_{i}=t_{i}-p<t_{i}$; if player $j \neq i$ wins the object at $\tau, x_{i}=e_{j i}<t_{i}$ by assumption. Hence $x_{i}<t_{i}$. Furthermore, $x_{2}+x_{3} \leq e_{12}+e_{13}$. Indeed, if player 1 wins the object at $\tau, x_{2}+x_{3}=e_{12}+e_{13}$. If, say, player 2 wins the object at $\tau$, the price $p$ must exceed $t_{1}-e_{21}$, otherwise player 1 would deviate from $\tau: x_{2}+x_{3}=t_{2}-p+e_{23} \leq t_{2}-t_{1}+e_{21}+e_{23} \leq e_{12}+e_{13}$, where the last inequality follows from (18). 
Let us $\operatorname{set}^{11}$

$$
y=\left(t_{1}-\epsilon, q x_{2}+(1-q) t_{2}, q x_{3}+(1-q) t_{3}\right)
$$

where $q$ is computed so that

$$
y_{1}+y_{2}+y_{3}=g_{\sigma}(N) \text {, i.e., } y_{2}+y_{3}=e_{12}+e_{13}
$$

namely

$$
q=\frac{\left(t_{2}+t_{3}\right)-\left(e_{12}+e_{13}\right)}{\left(t_{2}+t_{3}\right)-\left(x_{2}+x_{3}\right)}
$$

From the properties of $x_{2}$ and $x_{3}, q$ is well-defined and $0<q \leq 1$. We will show that $y \in C\left(g_{\sigma}\right)$. By construction, $y$ is efficient and individually rational. Let $S$ be a 2-player coalition. $g_{\sigma}(S)$ is the payoff of $S$ at the equilibrium $\zeta_{S} \equiv$ $\sigma(\{S, N \backslash S\})$ of the 2-player auction game in which $S$ competes against the singleton $N \backslash S$. It is easily checked that, at every equilibrium of an auction game with 2 players, the most efficient one wins the object (see, e.g., JM's proposition 2). Let $S=\{2,3\}$; by (18), player 1 wins the object at $\zeta_{\{2,3\}}$, so that $g_{\sigma}(\{2,3\})=e_{12}+e_{13}=y_{2}+y_{3}$. Let $S=\{1,2\}$; if player 3 wins the object at $\zeta_{\{1,2\}}, g_{\sigma}(\{1,2\})=e_{31}+e_{32} \leq t_{1}+e_{12}+e_{13}-t_{3} \leq y_{1}+y_{2}$, where the first inequality follows from (18) and the second one from $t_{3} \geq y_{3}+\epsilon$. If $\{1,2\}$ wins the object at $\zeta_{\{1,2\}}$, let $k=1$ or 2 be the most efficient player in $\{1,2\}$, i.e., $\max \left\{t_{1}+e_{12}, t_{2}+e_{21}\right\}=t_{k}+e_{k, k+1}$, where $k+1$ is $\bmod 2$. The price $p$ to be paid by $\{1,2\}$ at $\zeta_{\{1,2\}}$ must exceed $t_{3}-e_{k 3}$, otherwise player 3 would deviate from $\zeta_{\{1,2\}}$. Hence, $g_{\sigma}(\{1,2\}) \leq t_{k}+e_{k, k+1}-t_{3}+e_{k 3}$ so that $g_{\sigma}(\{1,2\}) \leq t_{1}+e_{12}+e_{13}-t_{3}$ by (18); the proof is completed as above. $S=\{1,3\}$ is similar.

\section{References}

[1] Arrow, K.J. (1979), "The property rights doctrine and demand revelation under incomplete information", M. Boskin (ed.), Economics and Human Welfare, Academic Press, New York.

\footnotetext{
${ }^{11}$ The idea is that the grand coalition, if it forms, allocates the object to the efficient player 1 . Then player 2 and player 3 must share $e_{12}+e_{13}$. Transfers are organized between these two players so that they get at least their individually rational level.
} 
[2] d'Aspremont, C. et L.-A. Gérard-Varet (1979), "Incentives and incomplete information", Journal of Public Economics 11, 25-45.

[3] d'Aspremont, C. et L.-A. Gérard-Varet (1982), "Bayesian incentive compatible beliefs", Journal of Mathematical Economics 10, 83-103.

[4] Aumann, R. (1961), "The core of a cooperative game without sidepayments", Transactions of the American Mathematical Society 98, 539552 .

[5] Bajari, P. (2001), "Comparing competition and collusion: a numarical approach", Economic Theory 18, 187-205.

[6] Barbar, R. and F. Forges (2007), "Collusion dans les enchères: quelques apports des jeux coopératifs", Revue Economique 58, 965-984.

[7] Biran, O. (2009), "Strategic partial collusion in auctions with externalities", mimeo, Université Paris-Dauphine.

[8] Caillaud, B. and P. Jehiel (1998), "Collusion in auctions with externalities", RAND Journal of Economics 29, 680-702.

[9] Forges, F., J.-F. Mertens and R. Vohra (2002), "The ex ante incentive compatible core in the absence of wealth effects", Econometrica 70, 18651892.

[10] Forges, F. and E. Minelli (2001), "A note on the incentive compatible core", Journal of Economic Theory 98, 179-188.

[11] Forges, F., E. Minelli and R. Vohra (2002), "Incentives and the core of an economy: a survey", Journal of Mathematical Economics 38, 1-41.

[12] Graham D., and R. Marshall (1987), "Collusive behavior at single-object second-price and English auctions", Journal of Political Economy 95, 1217-1239.

[13] Hafalir, I. (2007), "Efficiency in coalition games with externalities", Games and Economic Behavior 61, 242-258.

[14] Jehiel, P. and B. Moldovanu (1996), "Strategic non-participation", RAND Journal of Economics 27, 84-98. 
[15] Kalai, A. and E. Kalai (2009), "Engineering cooperation in two-player games", mimeo, Microsoft Research New England and Kellogg School of Management, Northwestern University.

[16] Krishna, V. (2002), Auction Theory, Academic Press.

[17] Lebrun, B. (1991), "Asymmetry in auctions", Ph.D. thesis, U.C.L.

[18] Lebrun, B. (1999), "First price auctions in the asymmetric n bidder case", International Economic Review 40, 125-142.

[19] Lucas, W. and Thrall, R. (1963), "n-person games in partition function form", Naval Research Logistics Quarterly 10, 281-298.

[20] Mc Afee R.P. and J. Mc Millan (1992), "Bidding rings", American Economic Review 82, 579-599

[21] Mailath, G. and P. Zemsky (1991), "Collusion in second price auctions with heterogeneous bidders", Games and Economic Behavior 3, 467486.

[22] Marshall, R. and L. Marx (2007), "Bidder collusion", Journal of Economic Theory 133, 374-402.

[23] Marshall, R., M. Meurer, J.-F. Richard and W. Stromquist (1994), "Numerical analysis of asymmetric first price auctions", Games and Economic Behavior 7, 193-220.

[24] Maskin, E. (2003): "Bargaining, coalitions and externalities," Presidential Address to the Econometric Society, Institute for Advanced Study, Princeton University.

[25] Myerson, R. (1984), "Cooperative games with incomplete information", International Journal of Game Theory 13, 69-86.

[26] Myerson, R. (2007), "Virtual utility and the core for games with incomplete information", Journal of Economic Theory 136, 260-285.

[27] Pesendorfer M. (2000), "A study of collusion in first-price auctions", Review of Economic Studies 67, 381-411. 
[28] Porter and Zona (1993), "Detection of bid rigging in procurement auctions", Journal of Political Economy 101, 518-538.

[29] Ray, D. (2007), A game-theoretic perspective on coalition formation, Oxford University Press.

[30] Ray, D. and R. Vohra (1997), "Equilibrium binding agreements", Journal of Economic Theory 73, 30-78.

[31] Waehrer, K. (1999), "Asymmetric auctions with application to joint bidding and mergers", International Journal of Industrial Organization $17,437-452$. 